\title{
HPLC/MS-based Metabolic Profiling and Quantification of Urine Free Amino Acids as Potential Biomarkers for Breast Cancer Diagnosis and Progression
}

\author{
Florina ROMANCIUC ${ }^{1)}$, Dan ENIU ${ }^{2)}$, Adelina STAICU'), Claudiu RACHIERU ${ }^{2)}$, Rares BUIGA ${ }^{2)}$, and Carmen \\ SOCACIU ${ }^{1,3 *)}$ \\ 1) University of Agricultural Sciences and Veterinary Medicine, 3-5 Mănăştur Street, Cluj-Napoca \\ 2) University of Medicine and Pharmacy "Iuliu Hațieganu” Cluj-Napoca \\ 3) RTD Center for Applied Biotechnology, in Diagnosis and Molecular Therapy, CCD-BIODIATECH Cluj- \\ Napoca, Romania; \\ *Corresponding author, e-mail: carmen.socaciu@usamvcluj.ro
}

Bulletin UASVM Animal Science and Biotechnologies 72(2) / 2015

Print ISSN 1843-5262; Electronic ISSN 1843-536X

DOI:10.15835/buasvmcn-asb:11588

\begin{abstract}
Considering the key role of free amino acids in cell metabolism and their predictive role as biomarkers in breast cancer by alteration of their metabolic pathways, the purpose of this study was to identify and quantify the urine amino acids from healthy and breast cancer women, diagnosed in different histological stages. Using advanced LC-QTOF-(ESI+)-MS technique and EZ:faast kit, based on derivatization of free aminoacids, 16 amino acids from control and breast cancer groups, preliminarily classified in I-IIIA and II-IIIB stages were identified and quantified. The general LC/MS fingerprint showed significant quantitative differences, especially for serine, lysine, tyrosine and leucine and asparagine, which decreased or increased, respectively. The multivariate analysis (PCA) showed good discrimination between normal and pathological groups, with best discrimination by essential amino acids. Cluster Analysis confirmed three discrimination regions, a major BC group, the control-group and a minor five patient-group. When the mean values of essential and non-essential amino acids were represented, in relation to cancer stages, gradual decreases of non-essential and essential amino acids were observed, from stage I to IIIA and from II to IIIB, in a parallel manner, non-essential amino acids having 3.5 times higher values than essential amino acids, the decreases being more relevant for non-essential amino acids. Serine had the steepest decrease, followed by Tyrosine, both being recommended as good biomarker candidates. By ROC curves, lysine and isoleucine showed highest AUC values and confidence intervals to provide good diagnostic between healthy and $\mathrm{BC}$ groups. Nonetheless, in agreement with quantitative data and correlations with the breast cancer stages, serine and tyrosine had better potential to offer a good diagnosis and confidence in discriminating among different cancer progression stages.
\end{abstract}

Keywords: urine amino acids, LC-QTOF-(ESI+)-MS, breast cancer, biomarker

\section{INTRODUCTION}

Cancer is the leading cause of death worldwide, breast cancer (BC) representing the most prevalent one in women, therefore new, rapid, sensitive and specific diagnostic methods are needed (Siegel et al., 2015). Recent advances in metabolomics have supported better understanding and enhanced clinical approach related to breast cancer early diagnosis and screening of potential biomarkers, able to discriminate among healthy and pathological samples, as well as indicate disease stages (Oakman et al., 2012). Global and focused (targeted) metabolomics are the two approaches employed for biomarkers discovery, both of them offering added value to disease prognosis and diagnosis (Zhang et al., 2014).

Amino acids are the main building blocks of proteins, with important roles as key metabolites 
and metabolism regulators, being considered central compounds of altered metabolic networks during tumor growth. Specific abnormalities of amino acid metabolism and levels have been reported in cancer, including breast cancer (Wise and Thompson, 2010; Nagata et al., 2014). The tumor's need for excessive consumption of amino acids for gaining energy (by carbohydrate and fat accumulation) results in a modification of amino acids concentration in blood (Fan et al., 2010, 2012). Some studies have reported decreased concentrations of amino acids in biofluids, but others have reported increased concentrations, the turnover being related to the type of cancer and specific metabolic pathways (Lai et al., 2005; Denkert et al., 2006; Kim et al., 2010; Mustafa et al., 2011; Poschke, et al., 2013; Armitage and Barbas, 2014; Gu et al., 2015). The amino acid profile in breast cancer patients was performed especially in blood serum and plasma (Vissers et al., 2005; Okamoto et al., 2009; Jobard et al., 2014), fewer being focused on excreted amino acids through urine (Slupsky et al., 2010).

The fact that a higher turnover of amino acids is a characteristic feature of the cancer cell, a decreased concentration of amino acids in urine is expected due to their upregulation by amino acid transporters, which determine the excessive consumption of blood amino acids from blood, and a decreased release of free amino acids in urine (Dankert et al., 2008).

Urine is an ideal bio-medium for disease study because it is readily available, easily obtained and less complex than other body fluids (due to its reduced content of protein and long-term storage without any changes) (Zhang et al., 2012; Bouatra et al., 2013). In recent years, increased research on urine metabolic profile by optimised protocols was reported, for different cancer types, from prostate (Shamsipur et al., 2013), to gastric (Fan et al., 2012), bladder (Kim et al., 2010), lung (Mathé et al., 2014), ovarian, cervical and breast cancer (Woo et al. 2009; Slupski et al., 2010; Soydinc et al., 2012).

The most frequently used analytical techniques in metabolomic studies, for amino acid fingerprinting and quantification, ready to be introduced also in clinical screeningare based ongas chromatography coupled with mass spectrometry (GC-MS), magnetic resonance spectrometry (MRS) but mostly, liquid chromatography with mass-spectrometry detection (LC-MS) (Patti et al., 2012; Arnald et al., 2015). The latest technique is used with or without preliminary derivatisation of amino acids, which became a more powerful technique due to its higher sensitivity (Wilson et al., 2011; Le et al., 2014).

This paper reports a targeted metabolomic procedure based on technique LC-QTOF-(ESI+)MS technique applied for derivatised amino acids, to investigate the profile and concentrations of individual free amino acids in urine of thirty breast cancer women (histologically classified in 5 stages) compared with five controls. By multivariate analysis (Principal Component Analysis and Cluster Analysis), the discriminations between the groups was done, identifying the amino acids as possible biomarkers for BC stagerelated diagnosis.

\section{MATERIALS AND METHODS}

Urine sample collection

The urine samples were collected from thirty breast cancer patients (BC), preliminarily diagnosed by histological examination, and classified in five stages ( I-IIIA and II-IIIB), according to international classification (http:// www.nationalbreastcancer.org), and compared with five control urine samples from healthy patients (C). The main characteristics of the patients' data are shown in Table 1.

\section{LC-QTOF-(ESI+)-MS analysis}

Volumes of $10 \mathrm{ml}$ of normal spot urine were collected in sterile containers. After homogenisation, the samples were centrifuged by 5 minutes at 2000 rotations per minute. After centrifugation, the supernatant was stored at $-20^{\circ} \mathrm{C}$ until analysis.

The amino acid fingerprinting and quantification was done by LC-QTOF-(ESI+)-MS technique and the protocol of EZ:faast amino acid free analysis kit as described previously by Romanciuc et al. (2014). The chromotographic separation was performed using a Thermo Scientific HPLC UltiMate 3000 system equipped with a quaternary pump delivery system Dionex and the mass spectra detection by a QTOF-(ESI+)MS Bruker Daltonics MaXis Impact device.

The column used for amino acid derivatisation was an EZ:faast AAA-MS (Phenomenex) (250x3.0 $\mathrm{mm}$ i.d.) using the solid phase extraction 
Tab.1. Clinical characterisation of the groups of patients, with similar BMI (Body Mass Index), preliminarily classified by histological criteria in different stages

\begin{tabular}{ccccc}
\hline Data set & Breast cancer (BC) & ID BC & Control (C) \\
\hline Number & 30 & - & 5 \\
\hline \multirow{2}{*}{ Age (Mean \pm SD) } & $57.53 \pm 12$ & - & $45 \pm 3$ \\
\hline BMI (Mean \pm SD) & $26.97 \pm 4$ & - & $26.56 \pm 7$ \\
\hline I A & 2 & BC8, BC18 & - \\
\cline { 2 - 5 } Stage & II A & 5 & BC11, BC13, BC17, BC20, BC23, BC26, BC29 & - \\
\cline { 2 - 5 } & III A & 7 & BC3, BC14, BC19, BC21, BC22, BC25, BC27, & - \\
\cline { 2 - 5 } & II B & 9 & BC6, BC9, BC10, BC12, BC15, BC24 & - \\
\cline { 2 - 5 } & III B & 6 & BC1 & - \\
\cline { 2 - 5 }
\end{tabular}

(SPE), a step followed by derivatisation. Three internal standard solutions were used, namely homoarginine (HARG), methionine- $\mathrm{D}_{3}\left(\mathrm{MET}_{3} \mathrm{D}_{3}\right)$ and homophenylalanine (HPHE) that are not found in urine. The derivatisation agent was propyl chloroformate.

For LC separation, the mobile phase consisted of $10 \mathrm{mM}$ ammonium formate in water (A) and $10 \mathrm{mM}$ ammonium formate in methanol (B). The flow rate was set at $0.250 \mathrm{ml} / \mathrm{min}$, and the column temperature was $35^{\circ} \mathrm{C}$. The gradient conditions used for the separation of amino acid derivatives were $68 \%$ B from 0 to 19 min followed by linear gradient to $93 \%$ B. The column was held at $93 \%$ B from 19.5 to $19.6 \mathrm{~min}$., then returned to initial conditions 68\% B from $19.6 \mathrm{~min}$ and allowed to equilibrate for $4.4 \mathrm{~min}$. Run-to-run time was $24 \mathrm{~min}$. The injected volume was $5 \mu \mathrm{L}$. Mass spectrometric conditions were: electrospray ion source (ESI) operated under positive ion mode, a nebulising gas pressure set at 2.8 bar, the drying gas flow at $12 \mathrm{~L} / \mathrm{min}$, the drying gas temp at $300^{\circ} \mathrm{C}$.

LC-MS data processing was done using successively the Base Peak Chromatogram, Dissect Chromatograms, Extracted Ion Chromatograms and QuantAnalysis (Bruker Daltonics software). Out of 27 derivatised amino acids, a number of 16 amino acids were quantified as presented in Table 2.

\section{Quantitative evaluation of amino acids and creatinine}

First, a calibration curve for creatinine was built and validated at a concentration range from 0.005 to $1 \mathrm{mg}$ creatinine $/ \mathrm{ml}$ (data not shown) and the mean correlation coefficient $\left(\mathrm{r}^{2}\right)$ for the curve was 0.96. All amino acid concentrations were calculated and expressed as nanomols per mg creatinine.

\section{Statistical analysis}

Multivariate data analysis was conducted using Mann-Whitney U tests for the group discrimination, Unscrambler X 10.1 (CAMO Software AS, Norway) for Principal Component Analysis and Cluster Analysis (PCA) and Cluster analysis (CA). For the ROC analysis, based on individual mean values of each amino acid in BC and control groups, the MedCalc 15.8 Software was applied (Ostend, Belgium) in order to demonstrate their possible utility as markers for early diagnosis of breast cancer. The quantitative data for individual, nonessential (NEAA) and essential (EAA) amino acids were calculated as mean \pm standard deviation (SD) values for both breast cancer (BC) and control (C) groups.

\section{RESULTS AND DISCUSSION}

Chromatographic amino acid separation and identification using Extracted Ion chromatogram (EIC).

From the initial base peak chromatogram obtained by LC-QTOF-(ESI+)-MS technique, including around 220 molecules (Fig.1 A and B), using Extracted Ion procedure, the EIC peaks corresponding to EAA and NEAA were selected, namely 16 derivatised amino acids identified and quantified (as presented in Table 2) eliminating other non-proteinogenic amino acids or derivatives. 


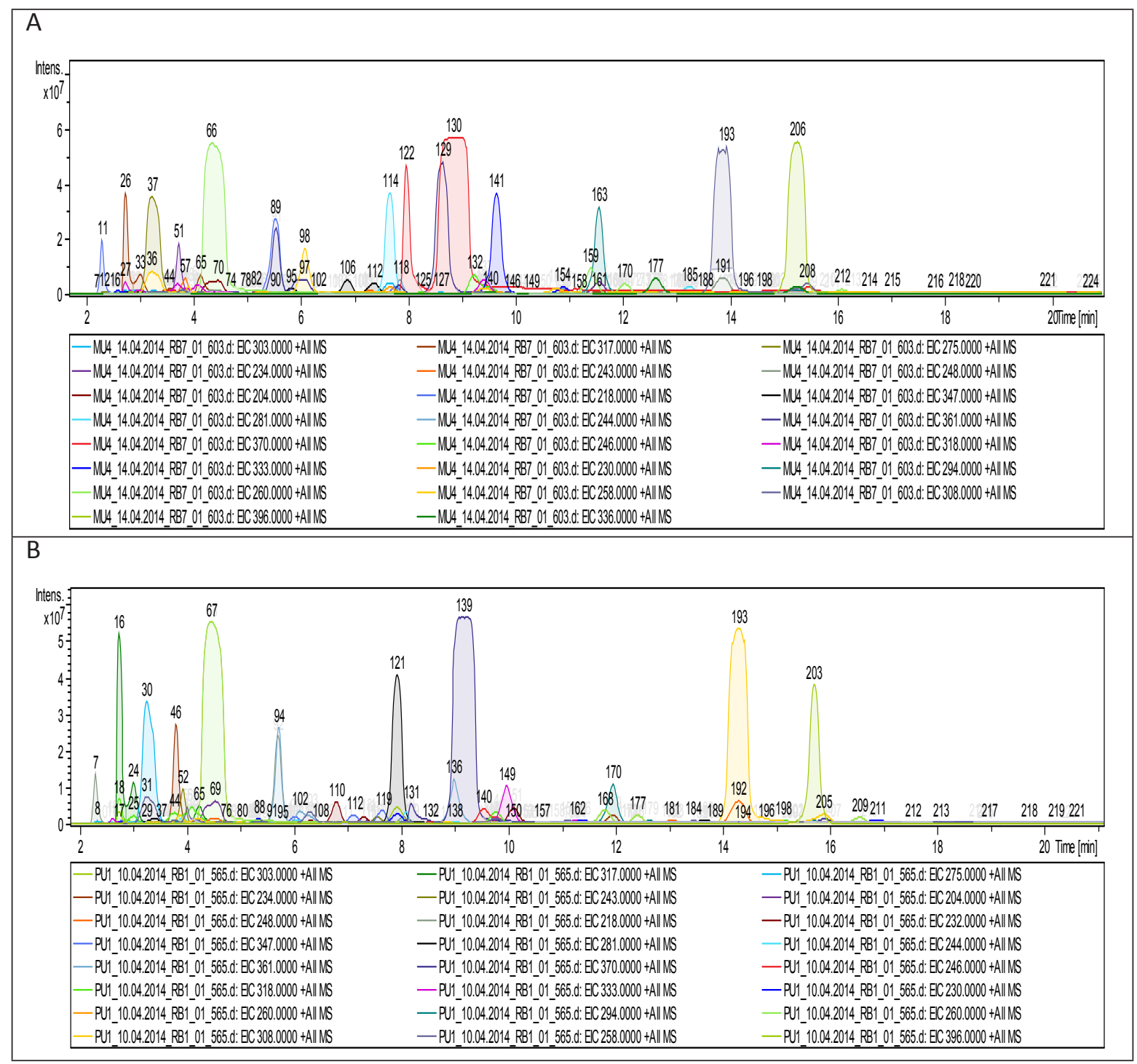

Fig.1. Comparative Extracted ion chromatograms (EIC) for derivatised amino acids separated from the control C (A) and BC (B) groups

All 16 amino acids quantified were identified in both groups. The general fingerprint was similar, the major differences were observed in lysine, arginine, valine, tryptophan, phenylalanine, tyrosine, which showed a decrease profile.

The identification of the amino acids separated by EIC (Fig.1A/1B), according to their peak number, $\mathrm{m} / \mathrm{z}$ value /molecular weight $\left(\left[\mathrm{M}+\mathrm{H}^{+}\right] /\right.$ MW) is presented in Table 2 .

\section{Amino acid quantitification}

The mean values of each amino acid (expressed in $\mathrm{nmol} / \mathrm{mg}$ creatinine) \pm standard deviations, for NEAA and EAA groups were calculated, as well as the ratios of values in $\mathrm{C}$ versus $\mathrm{BC}$ groups (C) $\mathrm{BC}$ ) and the statistical significance of differences (Table 3).

According to these data, one can notice general decreases of amino acid concentrations in BC group urines, in terms of total amino acids, as well essential (EAA) and non-essential (NEAA) ones. As individual concentrations, serine, glutamine, glycine, alanine and tyrosine were the major ones in the NEAA group (237-668 nmol/mg creatinine), while for the EAA group, lysine, hystidine and phenylalanine and tryptophan were major amino acids (from 119 to $270 \mathrm{nmol} / \mathrm{mg}$ creatinine) in 
Tab. 2. The identification of the amino acids separated by EIC( Fig.1A/ 1B), according to their peak number, $\mathrm{m} / \mathrm{z}$ value /molecular weight $\left(\left[\mathrm{M}+\mathrm{H}^{+}\right] / \mathrm{MW}\right) .{ }^{*}$ Internal standard

\begin{tabular}{cccccc}
\hline $\begin{array}{c}\text { Peak no. } \\
\text { Fig.1A/1B }\end{array}$ & {$\left[\mathrm{M}+\mathrm{H}^{+}\right] / \mathrm{MW}$} & Aminocid & $\begin{array}{c}\text { Peak no. } \\
\text { Fig.1A/1B }\end{array}$ & {$\left[\mathrm{M}+\mathrm{H}^{+}\right] / \mathrm{MW}$} & Aminocid \\
\hline $11 / 7$ & $303 / 174.2$ & Arginine & $130 / 139$ & $370 / 155.1$ & Histidine \\
\hline $26 / 16$ & $317 / 188.2$ & Homoarginine* & $132 / 140$ & $246 / 117.1$ & Valine \\
\hline $37 / 30$ & $275 / 146.2$ & Glutamine & $140 / 147$ & $318 / 147.1$ & Glutamic acid \\
\hline $51 / 46$ & $234 / 105.1$ & Serine & $141 / 149$ & $333 / 204.2$ & Tryptophan \\
\hline $57 / 52$ & $260 / 131.1$ & Asparagine & $159 / 169$ & $260 / 131.2$ & Leucine \\
\hline $78 / 80$ & $204 / 75.1$ & Glycine & $163 / 170$ & $294 / 165.2$ & Phenylalanine \\
\hline $89 / 94$ & $218 / 89.1$ & Alanine & $170 / 177$ & $260 / 131.2$ & Isoleucine \\
\hline $97 / 99$ & $347 / 132.1$ & Ornithine & $193 / 193$ & $308 / 179.2$ & Homophenylalanine* \\
\hline $114 / 121$ & $281 / 152.2$ & Methionine-d3* & $206 / 203$ & $396 / 181.2$ & Tyrosine \\
\hline $122 / 136$ & $361 / 146.1$ & Lysine & & \\
\hline \multicolumn{7}{c}{} \\
\hline
\end{tabular}

Tab. 3. Amino acid urine levels, expressed as mean values ( $\mathrm{x} \pm \mathrm{SD}, \mathrm{nmol} / \mathrm{mg}$ creatinine) in $\mathrm{BC}$ and Control (C) groups, their ratios, significance and tendency (increased or descreased mean values against controls)

\begin{tabular}{|c|c|c|c|c|c|c|c|}
\hline Category & Amino acid & Abr. & $\begin{array}{c}\mathrm{BC} \\
\text { nmol/mg } \\
\text { creatinine }\end{array}$ & $\begin{array}{c}\mathrm{C} \\
\text { nmol/mg } \\
\text { creatinine }\end{array}$ & $\begin{array}{l}\text { Ratio } \\
\text { C/BC }\end{array}$ & $\begin{array}{l}\text { Significance } \\
\text { (P-value) }\end{array}$ & $\begin{array}{c}\text { Tendency } \\
\text { BC/C }\end{array}$ \\
\hline \multirow{8}{*}{ 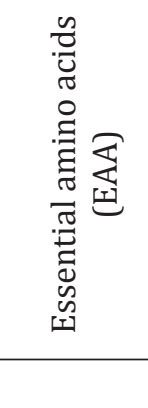 } & Histidine & His & $205.26 \pm 132$ & $229.4 \pm 93$ & 1.11 & $\mathrm{P}>=0.05$ & Slight decrease \\
\hline & Leucine & Leu & $27.5 \pm 17$ & $14.73 \pm 5$ & 0.53 & $\mathrm{P}=0.05$ & Strong Increase \\
\hline & Isoleucine & Ile & $27.77 \pm 16$ & $49.75 \pm 17$ & 1.79 & $\mathrm{P}=0.01$ & Decrease \\
\hline & Lysine & Lys & $89.78 \pm 73$ & $270.12 \pm 60$ & 3.03 & $\mathrm{P}=0.001$ & Strong decrease \\
\hline & Phenilalanine & Phe & $85.53 \pm 45$ & $139.5 \pm 80$ & 1.62 & $\mathrm{P}>=0.05$ & Decrease \\
\hline & Tryptophan & Tyr & $82.34 \pm 48$ & $119.69 \pm 58$ & 1.45 & $P>=0.05$ & Decrease \\
\hline & Valine & Val & $53.71 \pm 27$ & $75.7 \pm 26$ & 1.4 & $\mathrm{P}>=0.05$ & Dercrease \\
\hline & Total EAA & & 571.92 & 898.92 & 1.57 & $P>=0.05$ & DECREASE \\
\hline \multirow{11}{*}{ 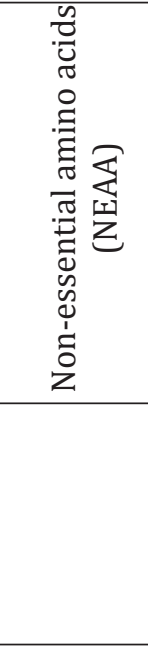 } & Alanine & Ala & $237.36 \pm 135$ & $310.21 \pm 113$ & 1.3 & $\mathrm{P}>=0.05$ & Decrease \\
\hline & Arginine & Arg & $18.62 \pm 12$ & $27.04 \pm 10$ & 1.45 & $\mathrm{P}>=0.05$ & Decrease \\
\hline & Asparagine & Asn & $29.35 \pm 72$ & $22.73 \pm 11$ & 0.77 & $P>=0.05$ & Increase \\
\hline & Glutamine & Gln & $388.58 \pm 61$ & $567.89 \pm 190$ & 1.46 & $P>=0.05$ & Decrease \\
\hline & Glutamic acid & Glu & $14.92 \pm 9$ & $17.38 \pm 12$ & 1.16 & $P>=0.05$ & Slight Decrease \\
\hline & Glycine & Gly & $371.78 \pm 190$ & $498.39 \pm 330$ & 1.34 & $P>=0.05$ & Decrease \\
\hline & Serine & Ser & $418.64 \pm 245$ & $668.33 \pm 199$ & 1.59 & $\mathrm{P}=0.01$ & \multirow{2}{*}{ Strong decrease } \\
\hline & Tyrosine & Tyr & $122.82 \pm 76$ & $203.48 \pm 81$ & 1.66 & $\mathrm{P}=0.04$ & \\
\hline & Total NEAA & & 1602.11 & 2315.49 & 1.44 & $P>=0.05$ & DECREASE \\
\hline & $\begin{array}{c}\text { Total AA } \\
(\text { EAA+NEAA) }\end{array}$ & & 2174.022 & 3214.402 & 1.48 & $\mathrm{P}>=0.05$ & DECREASE \\
\hline & $\begin{array}{c}\text { Ratio NEAA/ } \\
\text { EAA }\end{array}$ & & 2.80 & 2.57 & 0.91 & & \\
\hline Other & Ornitine & Orn & $16.38 \pm 10$ & $16.69 \pm 3$ & 1.01 & $P>=0.05$ & - \\
\hline
\end{tabular}




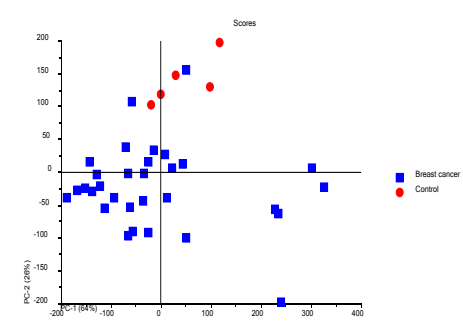

EAA

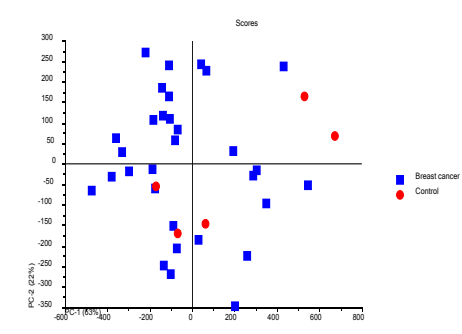

NEAA

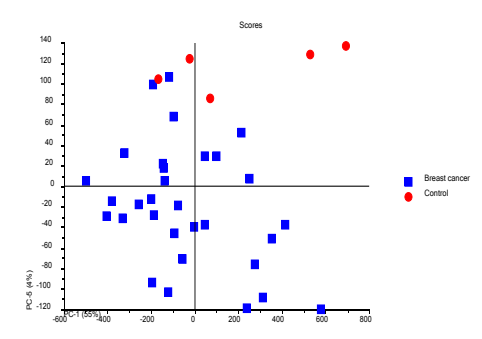

Total AA

Fig. 2. Comparative PCA-scores which allow the discrimination between the control group and Breast cancer groups, independent of tumor stage, considering EAA, NEAA and total aminoacid concentrations

$\mathrm{C}$ group, while in $\mathrm{BC}$ group, the mean values decreases around 35\%.

Looking at individual amino acids, in the EAA group, all concentrations decreased except Leu. Strong and significant decrease against control was noticed for Lys (ratio $\mathrm{C} / \mathrm{BC}=3.03$, $\mathrm{P}=0.001)$, while for Leu, an increased ratio $(\mathrm{C} /$ $\mathrm{BC}=0.53$ ), and opposite to isoleucine which decreased $(C / B C=1.79)$. In the NEAA group, also, except asparagine $(\mathrm{C} / \mathrm{BC}=0.77)$, all amino acid concentrations decreased, more significantly for serine and tyrosine $(\mathrm{C} / \mathrm{BC}=1.59$ and 1.66, respectively).

These data are well correlated with specific amino acid pathways. It is known that out of the 20 amino acids found in proteins, around 50\% are NEAA, and the others (EAA) are supplied by the diet. Each amino acid undergoes its own metabolism and performs specific functions, so it is very difficult to trace their individual turnover. Nevertheless, free amino acid turnover is easier to follow, especially for some major representatives like Glu and Gln, which represents up to $50 \%$ from NEAA, in our case $25 \%$ in both BC and C groups. Since free amino acids are not stored but undergo different catabolic modifications by oxidative or non-oxidative deamination, transamination, urea cycle, leading to intermediates (such as Acetyl CoA) for carbohydrate and fatty acids/ sterols biosynthesis. In this context, the cancer cells are consuming larger quantities of aminoacids, needed for their energetic survival, leaving lower quantities in biofluids, such as blood, but especially released in urine. Finally, this is a general explanation for decreased concentrations of almost all amino acids in BC patients. Of course, a deeper discussion is needed, to explain which are the specific pathways where the five amino acids
(Lys, Leu, Asn, Tyr and Ser) showed significant modifications.

Lower concentrations of other urine amino acids in cancer patients were reported by other authors, such as alanine, glutamine, glycine, tryptophan, phenylalanine and valine, or methionine, isoleucine, phenylalanine and arginine (Okamoto et al., 2009), our results being consistent with these studies and others, mainly made on serum and plasma (Proenza et al., 2003; Vissers et al., 2005), fewer on urine ( Slupsky et al., 2010; Miyagi et al., 2011; Barnes et al., 2014).

\section{Multivariate analysis}

Based on the quantitative data presented above (for the EAA, NEAA and TAA groups) for BC and Controls, the Principal Component Analysis (PCA) was performed, in order to discriminate differences among these groups, as presented in Fig 2.

The PCA graphics shows good discrimination between normal and pathological groups based on amino acid category, a very good discrimination when EAA were considered (PC1-64\%), and lower discrimination potential where total amino acids and NEAA were considered (PC1-55\% and $63 \%$, respectively). In conclusion, the best discrimination was obtained between $\mathrm{BC}$ and controls, when EAA were considered.

For a better view of grouping derived from PCA-EAA (Fig. 2, left) , Cluster Analysis (as shown in Fig.3) confirmed three discriminating regions: a general BC group including 25 samples (below), a control-group $(\mathrm{C})$ clustered in the middle and another small group of 5 BC samples (above). Further investigation of the characteristics of the 5 samples is needed. 


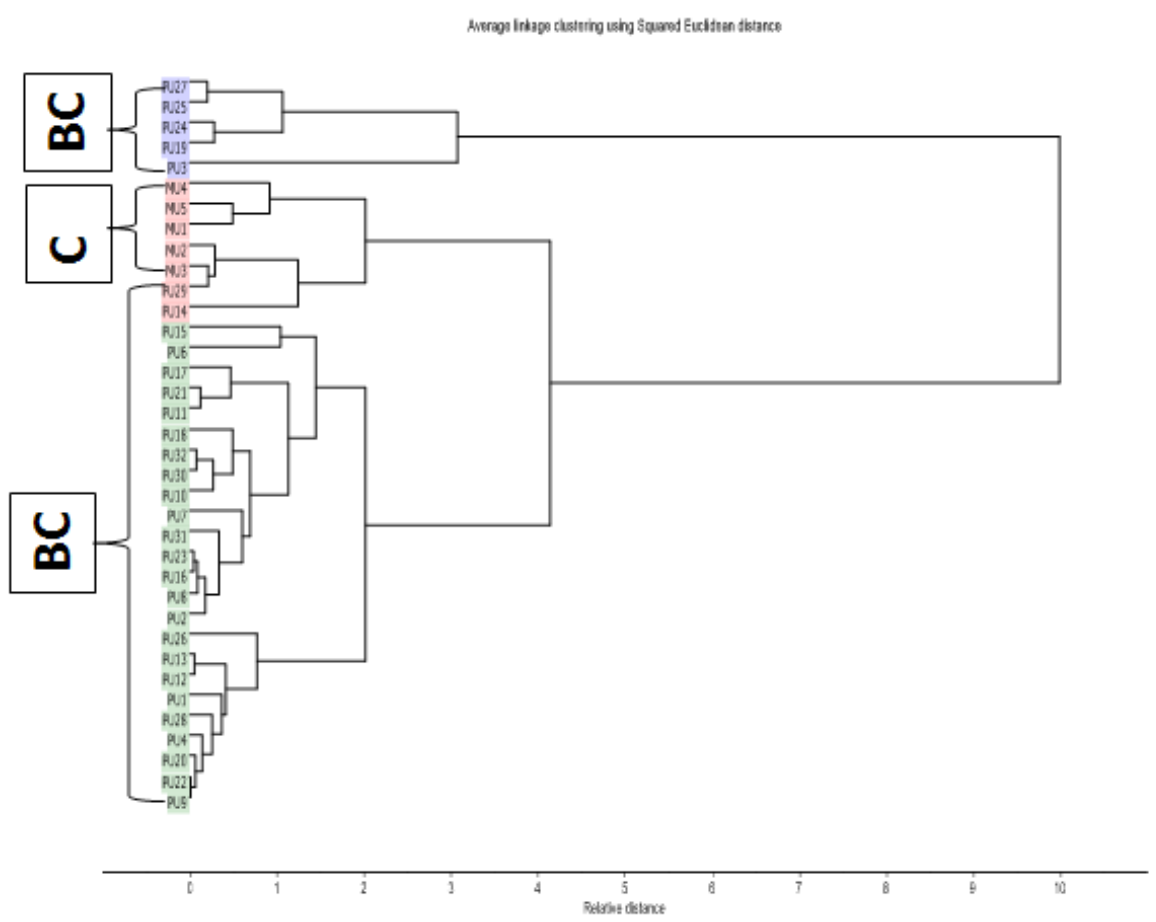

Fig. 3. Cluster analysis (CA), based on the Euclidian distances scale, for all 35 subjects, considering mean values of individual urine EAA as a complement of PCA analysis.
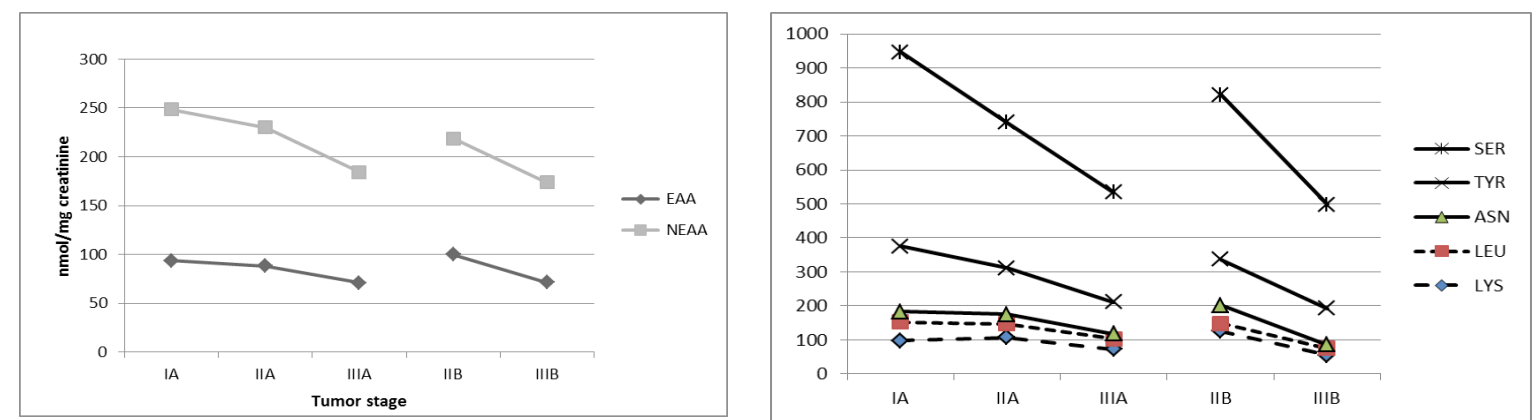

Fig. 4. Left: Correlations between the tumor stages (I-IIIA, II-IIIB) and the mean values of Essential (EAA) and non-essential amino acids (NEAA). Right: same correlations considering mean values of Lys, Leu (EAA), Asn, Ser and Tyr (NEAA).

Dependence of amino acid concentrations on the $B C$ tumor stage

In order to see the relevance of EAA, NEAA and individual amino acid concentrations for the tumor progression stage, and possible statistical correlations, the mean values of the sum of EAA, NEAA and the five amino acids with significant differences against controls, depending on the tumor stage, were represented graphically.

The main reason to see the dependence of amino acid concentrations on the BC tumor stage is the possibility to predict, from the individual or group levels of amino acids, the stage of tumor progression, as reported in other studies, but using plasma amino acids (Okamoto et al., 2009).

To have a better image of amino acid profile related to the different stages (IA-IIA and IIB-IIIB), mean values of total essential (EAA) and nonessential amino acids (NEAA) were represented (Fig. 4-left); the mean values for the five amino acids (Ser, Tyr, Asn, Leu and Lys) (Fig. 4-right), in relation with their absolute values (presented in Table 3) from all tumor stages are presented comparatively.

According to Fig.4-left, one can see gradual decreases of NEAA and EAA, from stage I to III (A) 
and from II to IIIB, in a parallel manner, the NEAA having aprox. 3.5 times higher values than EAA. Comparatively, the modifications are more relevant in the NEAA group.

According to Fig. 4-right, Ser (the major amino acid) had the steepest decrease, followed by Tyr, related to the tumor stage and can be considered therefore good potential biomarkers. Between stage I-II A no differences are found, and there is a slow decrease between II and IIIA for the other amino acids ( Asn, Leu and Lys). Comparatively, the decrease from stage II to IIIB is more significant.

Therefore, considering similar observations (Myiagi et al., 2011), the decrease of urine Serine and Tyrosine, as major NEAA, in early stages ( from IA to IIA or IIB and then IIIA or IIB) is a good reason to consider them as potential good biomarkers, significantly related to tumor progression.

\section{ROC Curves}
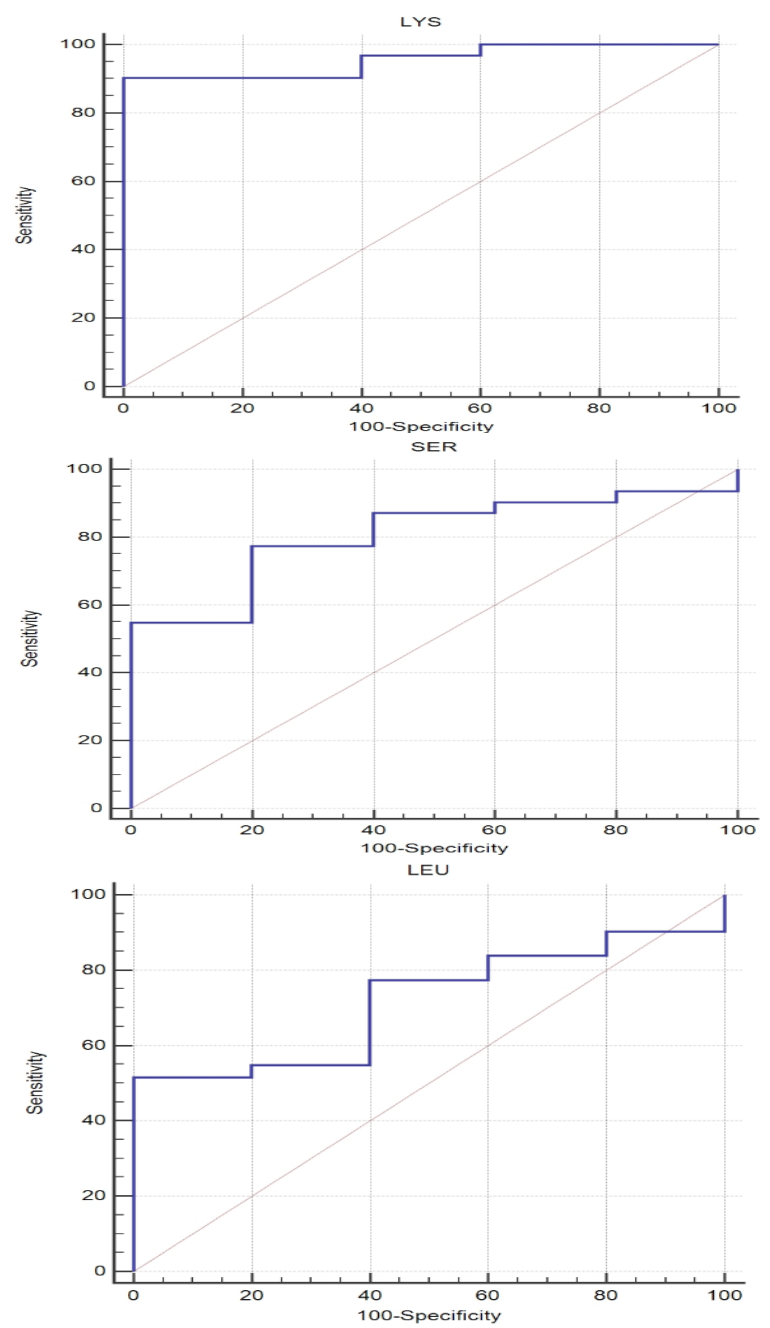

To validate these five individual aminoacids, including also the isomer of Leu, Ileu, the test by Reciver Opereting Characteristic Curve (ROC) was carried out, as presented in Fig 5.

The ROC curves of the cross-validated predicted class values were made for the individual amino acids which were identified to have significant modifications against controls ( $p$ $<0.5$ ).

The good prediction as biomarker is related to higher AUC values, sensitivity and specificity. As presented in Fig.5, AUC values (with 95\% confidence interval-CI) were:

for Lys $=0.95$, sensitivity $100 \%$, specificity $90.3 \%$,

CI from 0.82 to 0.99

for ILeu $=0.84$, sensitivity $74.19 \%$, specificity $100 \%$, CI from 0.68 to 0.94

for Ser $=0.80$, sensitivity $77.42 \%$, specificity $80 \%$, CI from 0.64 to 0.91
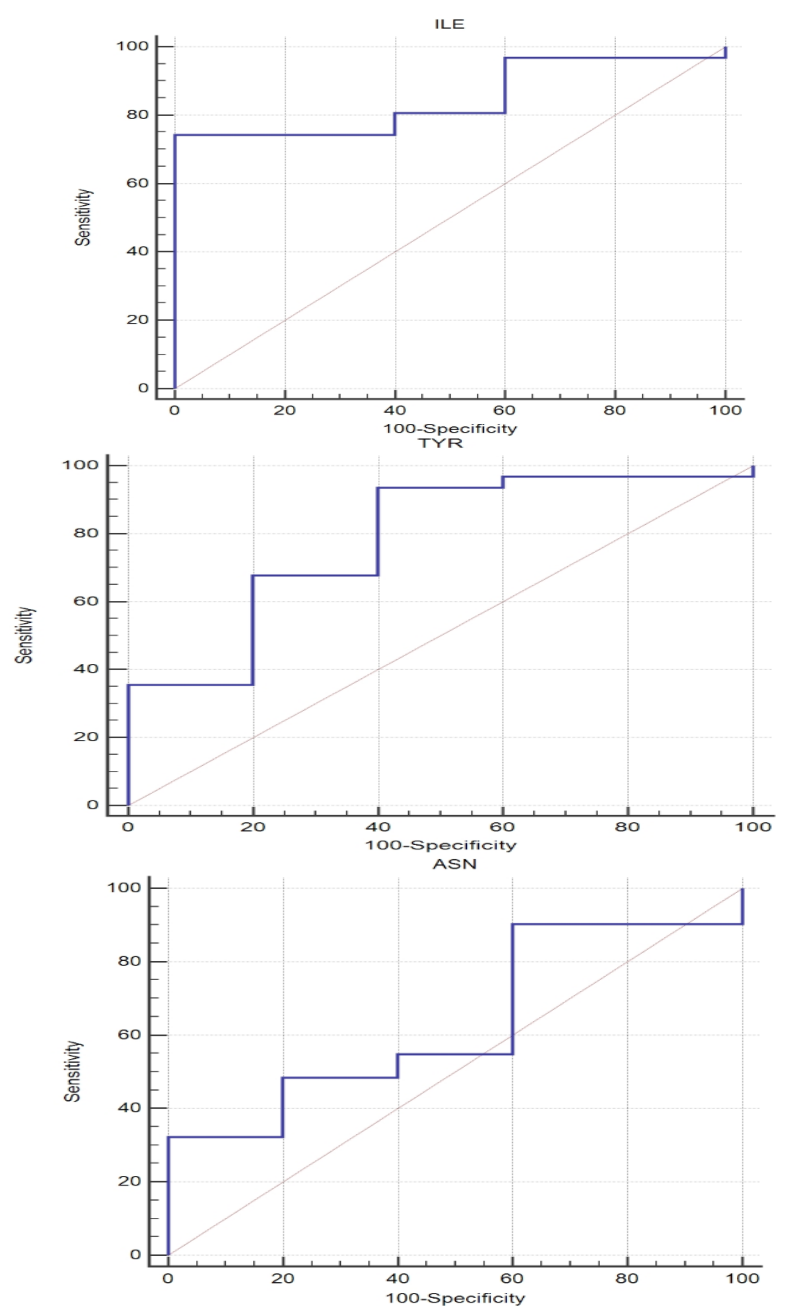

Fig. 5. ROC representation of tumor prognostic for six amino acids, as potential urine biomarkers (Leu, Ile, Lys, Ser, Asn, Ser and Tyr) for breast cancer 
for Tyr $=0.78$, sensitivity $93.55 \%$, specificity $60 \%$, $\mathrm{CI}$ from 0.61 to 0.90

for Leu $=0.71$, sensitivity $51.61 \%$, specificity $100 \%$, CI from 0.54 to 0.85

for Asn $=0.63$, sensitivity $32.26 \%$, specificity $100 \%$, CI from 0.45 to 0.78

According to ROC parameters, Lys and Ileu showed highest AUC values and highest 95\% confidence intervals to make good diagnostic between healthy and BC groups, while Ser and Tyr had slightly smaller AUC values, but a better potential to offer a good diagnostic with good confidence, in discriminating among different $\mathrm{BC}$ progression stages. Leu and Asn had inferior predictibility value, they actually increased in BC group and had an opposite feature. This finding suggested that all four amino acids, namely Lys, Ileu, Ser and Tyr have a good predictibility potential to be considered urine biomarkers for breast cancer diagnosis and tumor progression. A statistically significant decrease of Lysine was also signalled in a study conducted by Barnes et al. (2014).

\section{CONCLUSION}

Using targeted metabolomics, an optimized method to identify and quantify urine free amino acids as potential biomarkers for breast cancer and correlation to its stages was performed.

Using advanced LC-QTOF-(ESI+)-MS technique and the specific advanced EZ:faast amino acid free analysis kit based on derivatisation of free aminoacids, 16 urinary amino acids from control and breast cancer groups of patients were identified and quantified, preliminarily classified by histology in I-IIIA and II-IIIB stages. The general fingerprint of extracted Ion chromatograms was similar, with quantitative differences, especially for five amino acids: serine, lysine, isoleucine, tyrosine and asparagine, which generally showed decreases, but also increases (for asparagine and leucine).

The multivariate analysis, as seen in the PCA graphics, shows good discrimination between normal and pathological groups, with best discrimination by EAA (PC1-64\%), and lower discrimination potential by total amino acids and NEAA (PC1-55\% and 63\%, respectively). Cluster Analysis confirmed three discriminating regions, a major BC group of 25 samples, the control-group and a five BC patient-group. Further investigation of the characteristics of the 5 samples is needed.
To have a better image of amino acid profile related to the different stages (I-IIA and II-IIIB), the mean values of essential and non-essential amino acids were represented also for the five amino acids mentioned above.

There were observed gradual decreases of non-essential and essential amino acids, from stage I to IIIA and from II to IIIB, in a parallel manner, non-essential amino acids having 3.5 times higher values than EAA. The decreases were more relevant for non-essential amino acids. Considering individual amino acid dynamic, Serine had the steepest decrease, followed by Tyrosine and best related to the tumor stage, so they are good biomarker candidates. Comparatively, the decrease from stage II to IIIB is more significant.

According to ROC curves, the aminoacids Lysine and Isoleucine showed highest AUC values and highest 95\% confidence intervals to make good diagnostic between healthy and $\mathrm{BC}$ groups. Meanwhile, in agreement with quantitative data and correlations with the breast cancer stages, Serine and Tyrosine had better potential to offer a good diagnosis and confidence in discriminating among different $\mathrm{BC}$ progression stages, while Leucine and Asparagine, which increased in the breast cancer group, had inferior predictibility values.

In conclusion, from the analysed urine samples, it is suggested that four free urine amino acids have a good diagnosis prediction value, namely Lys and Ileu, to discriminate between healthy and breast-cancer patients, while Ser and Tyr, have high predictibility potential to be considered diagnosis biomarkers for different stages of breast cancer progression.

\section{REFERENCES}

1. Armitage EG, Barbas C (2014). Metabolomics in cancer biomarker discovery: Current trends and future perspectives. Journal of Pharmaceutical and Biomedical Analysis 87:1-11.

2. Arnald A, Marsal S, Julià A (2015). Analytical methods in untargeted metabolomics: state of the art in 2015. Front. Bioeng. Biotechnol. 3:23.

3. Barnes T, Bell K, DiSebastiano KM, Vance V, Hanning R, Russell C, Dubin JA, Bahl M, Califaretti N, Campbell C, Mourtzakis M (2014). Plasma amino acid profiles of breast cancer pacients early in the trajectory of the disease differ from healthy comparison groups. Appl. Physiol. Nutr. Metab. 39:740-744.

4. Beger RD (2013). A review of applications of metabolomics in cancer. Metabolites 3:552-574.

5. Bouatra S, Aziat F, Mandal R, Guo AC, Wilson MR, Knox C, Bjorndahl TC, Krishnamurthy R, Saleem F, Liu P, Dame ZT, Poelzer J, Huynh J, Yallou FS, Psychogios N, Dong E, 
Bogumil R, Roehring C, Wishart DS (2013). The Human Urine Metabolome, PLOS ONE 8:e73076.

6. Denkert C, Budczies J, Kind T, Weichert W, Tablack P, Sehouli J, Niesporek S, Konsgen D, Dietel M, Fiehn O (2006). Mass spectrometry-based metabolic profiling reveals different metabolite patterns in invasive ovarian carcinomas and ovarian borderline tumors. Cancer Res. 66:10795-10804.

7. Dankert C, Budczies J, Weichert W, Wohlgemuth G, Scholz M, Kind T, Niesporek S, Noske A, Buckendahl A, Dietel M, Fiehn O (2008). Metabolite profiling of human colon carcinoma - deregulation of TCA cycle and amino acid turnover. Molecular Cancer 7:72-78.

8. Fan J, Hong J, Hu JD, Chen JL (2012). Ion chromatography based urine amino acid profiling applied for diagnosis of gastric cancer. Gastroenterology Research and Practice, Article ID 474907.

9. Fan Y, Wang J, Yang Y, Liu Q, Fan Y, Yu J, Zheng S, Li M, Wang J (2010) Detection and identification of potential biomarkers of breast cancer, J. Cancer Res. Clin. Oncol., 136:1243-1254.

10. Gu Y, Chen T, Fu S, Sun X, Wang L, Wang J, Lu Y, Ding S, Ruan G, Teng L, Wang M (2015). Perioperative dynamics and significance of amino acid profile in patients with cancer. Journal of Translational Medicine 13:35.

11. Jobard E, Pontoizeau C, Blaise BJ, Bachelot T, Herrmann BE, Tredan O (2014). A serum nuclear magnetic resonancebased metabolomic signature of advanced metastatic human breast cancer. Cancer Letters, 343: 33-41.

12. Kim JW, Lee G, Moon SM, et al. (2010). Metabolomic screening and star patters recognition by urinary amino acid profile analysis from bladder cancer patients. Metabolomics, 6:202-206.

13. Lai HS, Lee JC, Lee PH, Park MJ, Hong SK, Ahn YH,. Kim KR, Paik MJ (2005). Plasma free amino acid profile in cancer patients. Seminars in Cancer Biology 15:267-276.

14. Le A, Ng A, Kwan T, Cusmano-Ozog K, Cowan TM (2014). A rapid, sensitive method for quantitative analysis of underivatized amino acids by liquid chromatographytandem mass spectrometry (LC-MS/MS). Journal of Chromatography B 944: 166-174.

15. Mathé EA, Patterson AD, Haznadar M, Manna SK, Krausz KW, Bowman ED, Shields PG, Idle JR, Smith PB, Anami K, Kazandjian DG, Hatzakis E, Gonzalez FJ, Harris CC (2014) Non-invasive urinary metabolomic profiling identifies diagnostic and prognostic markers in lung cancer. Cancer Res. 74(12):3259-70.

16. Miyagi Y, Igashiyama M, Gochi A, Akaike M, Ishikawa T, Miura T, Saruki N, Bando E, Kimura H, Imamura F, Moriyama M, Ikeda I, Chiba A, Oshita F, Imaizumi A, Yamamoto H, Miyano H, Horimoto K, Tochikubo O, Mitsushima T, Yamakado M, Okamoto N (2011). Plasma free amino acid profiling of five types of cancer patients and its application for early detection. Plos One, 6(9): e24143.

17. Mustafa A, Gupta S, Hudes GR, Egleston BL, Uzzo RG, Kruger WD (2011). Serum amino acid levels as a biomarker for renal cell carcinoma. J. Urol. 186(4):1206-1212.

18. Nagata C, Wada K, Tsuji M, Hayashi M, Takede N, Yasuda K (2014). Plasma amino acids profiles are associate with biomarkers oof breast cancer risk in premenopausal Japanese women. Cancer Causes Control 25:143-149.

19. Oakman C, Tenori L, Cappadona SS, Luchinat C, Bertini I, Di Leo A (2012). Targeting metabolomics in breast cancer. Current Breast Cancer Reports, 4 (4): 249-256.
20. Okamoto N, Miyagi Y, Chiba A, Akaike M, Shiozawa M, Imaizumi A, Yamamoto H, Ando T, Yamakado M, Tochikubo 0 (2009). Diagnostic modeling with differences in plasma amino acid profiles between non-cachectic colorectal/breast cancer patients and healthy individuals. International Journal of Medicine and Medical Sciences 1(1): 001-008.

21. Patti GJ, Yanes O, Siuzdak G (2012). Innovation: Metabolomics: the apogee of the omics trilogy. Nat. Rev. Mol. Cell Biol.13(4): 263-9.

22. Poschke I., Mao Y, Kiessling R, Boniface J (2013). Tumordependent increase of serum amino acid levels in breast cancer patients has diagnostic potential and correlates with molecular tumor subtypes. Journal of Translational Medicine, 11:290-298.

23. Proenza AM, Oliver J, Palou A, Roca P (2003). Breast and lung cancer are associated with a decrease in blood cell amino acid content. Journal of Nutritional Biochemistry 14:133-138.

24. Romanciuc EF, Pop R, Socaciu C (2014). UPLC-QTOF (ESI +) MS Analysis Applied for Targeted Profiling of Human Urine Amino Acids. Bulletin USAMV Animal Science and Biotechnologies 71(2), 250-255.

25. Shamsipur M, Naseri MT, Babri M (2013). Quantification of candidate prostate cancer metabolite biomarkers in urine using dispersive derivatization liquidliquid microextraction followed by gas ans liquid chromatography-mass spectrometry. Journal of Pharmaceutical and Biomedical Analysis, 81-82: 65-75.

26. Slupsky CM, Steed H, Wells TH, Dabbs K, Schepansky A, Capstick V, Faught W, Sawyer MB (2010). Urine metabolite analysis offers potential early diagnosis of ovarian and breast cancers. Clin. Cancer Res 1;16(23):5835-41.

27. Soydinc HO, Duranyildiz D, Guney N, Derin D, Yasasever V (2012). Utility of serum and urine upar levels for diagnosis of breast cancer. Asian Pacific Journal of Cancer Prevention, 13 (6):2887-2889.

28. Vissers YLJ, Dejong HC, Luiking Y, Fearon KCH, Meyenfeldt MF, Deutz NEP (2005), Plasma arginine concentrations are reduced in cancer patients: evidence for arginine deficiency?.American J Clinical Nutrition 81:1142-6.

29. Wilson SF, James CA, Zhu X, Davis TM, Rose MJ (2011). Development of a method for the determination of glycine in human cerebrospinale fluid using pre-column derivatization and LC-MS/MS. Journal of Pharmaceutical and Biomedical Analysis 56: 315-323.

30. Wise DR and Thompson CR, 2010, Glutamine Addiction: A New Therapeutic Target in Cancer, Trends Biochem Sci. 35:427-433.

31. Woo HM, Kim KM, Choi MH, Jung BH, Lee J, Kong G, Nam SJ, Kim S, Bai SW, Chung BC (2009). Mass spectrometry based metabolomic approaches in urinary biomarker study of women's cancer. Clinica Chimica Acta 400(1-2): 63-69.

32. Zhang A., Sun H, Wu X, Wang X (2012). Urine metabolomics, Clinica Chimica Acta 414:65-69.

33. Zhang A, Sun H, Yan G, Wang P, Wang X (2014). Metabolomics for biomarker discovery: Moving to the clinic. BioMed Research International 174: 6-12.

34. http://www.nationalbreastcancer.org 